\title{
Neurapraxia in patients with trigeminal neuralgia but no identifiable neurovascular conflict during microvascular decompression: a retrospective analysis of 26 cases
}

Juan $\mathrm{Li}^{1 \dagger}$, Min Zhou ${ }^{2 \dagger}$, Yuhai Wang ${ }^{3}$, Sze Chai Kwok ${ }^{4,5,6}$ and Jia Yin ${ }^{1 *}$

\begin{abstract}
Background: Microvascular decompression (MVD) is the first choice in patients with classic trigeminal neuralgia (TGN) that could not be sufficiently controlled by pharmacological treatment. However, neurovascular conflict (NVC) could not be identified during MVD in all patients. To describe the efficacy and safety of treatment with aneurysm clips in these situations.

Methods: A total of 205 patients underwent MVD for classic TGN at our center from January 1, 2015 to December 31, 2019. In patients without identifiable NVC upon dissection of the entire trigeminal nerve root, neurapraxia was performed using a Yasargil temporary titanium aneurysm clip (force: $90 \mathrm{~g}$ ) for $40 \mathrm{~s}$ (or a total of $60 \mathrm{~s}$ if the process must be suspended temporarily due to bradycardia or hypertension).

Results: A total of 26 patients (median age: 64 years; 15 women) underwent neurapraxia. Five out of the 26 patients received prior MVD but relapsed. Immediate complete pain relief was achieved in all 26 cases. Within a median followup of 3 years (range: 1.0-6.0), recurrence was noted in 3 cases (11.5\%). Postoperative complications included hemifacial numbness, herpes labialis, masseter weakness; most were transient and dissipated within 3-6 months.

Conclusions: Neurapraxia using aneurysm clip is safe and effective in patients with classic TGN but no identifiable NVC during MVD. Whether this method could be developed into a standardizable method needs further investigation.
\end{abstract}

Keywords: Trigeminal neuralgia, Microvascular decompression, Offending vessel, neurapraxia

\section{Background}

Microvascular decompression (MVD) is the first choice in patients with classic trigeminal neuralgia (TGN) that could not be well controlled by pharmacological treatments $[1,2]$. However, in $3.1-28.8 \%$ of the cases, offending vessels (OVs) could be identified during MVD [3,

\footnotetext{
*Correspondence: jianyiyin@hotmail.com

†Juan Li and Min Zhou contributed equally to this work

1 Department of Neurosurgery, Shanghai Tenth People's Hospital, Tongji

University, No. 301 Yanchang Road, Shanghai 200072, China

Full list of author information is available at the end of the article
}

4]. In such cases, second surgical operation that ablates the extracranial segment of the trigeminal nerve may be needed. Revuelta-Gutierrez et al. reported promising results of neurapraxia with bipolar tips during MVD in such patients [5]. In 2014, we attempted to develop a standardizable method of neurapraxia by using a Yasargil temporary titanium aneurysm clip (force: $90 \mathrm{~g}$ ). In the initial series of 3 cases, the sensory root or the main trunk of the trigeminal nerve was clipped for $2.5 \mathrm{~min}$, but we observed severe hemifacial numbness and masseter weakness in all 3 cases. Starting from the beginning of 
2015, we decreased the clipping duration to $40 \mathrm{~s}$. In this retrospective analysis, we analyzed the data of all cases with at least 1-year follow-up.

\section{Methods}

The current study was conducted in compliance with the principles outlined in the Declaration of Helsinki, and was approved by the Ethics Committee of Shanghai Tenth People's Hospital (approval \#: CPPRB1). Informed consent was waived due to the nature of the study. All patient data were anonymized in the paper. We retrospectively screened all cases of MVD for TGN at our center during a period from January 1, 2015 to December 31, 2019. The diagnosis of TGN was established based on the criteria by the International Classification of Headache Disorders 3 (ICDH-3; 13.1.1.1). All subjects received MR-angiography prior to the surgery. MVD was performed using a standard suboccipital retrosigmoid approach. After releasing cerebrospinal fluid (CSF) under the microscope, the cerebellar hemisphere was retracted, and the arachnoid membrane between the petrosal vein and the facial-auditory nerve was opened to expose the cisternal segment of the trigeminal nerve. The entire length of the trigeminal nerve root (from the pons to the entrance of Meckel's cave) was dissected to identify NVC. Teflon fragments were applied between the trigeminal nerve and offending vessels.

In cases without identifiable NVC, neurapraxia was conducted using a standard straight Yasargil temporary titanium aneurysm clip (Aesculap-B. Braum, Germany; $90-\mathrm{g}$ force) for $40 \mathrm{~s}$, preferably to the sensory root (Fig. 1), or the main trunk of the trigeminal nerve if sensory root was not separate from the motor root (Fig. 2). The procedure could be readily conducted in patients with recurrent pain after previous MVD (Fig. 3). In subjects with clinically significant bradycardia or hypertension during neurapraxia, the aneurysm clip was released temporarily and the process was repeated to achieve 60-s accumulative time for neurapraxia before. Sutures were removed 7 days later. The last follow-up (via either office visit or telephone) was conducted in December 2020.

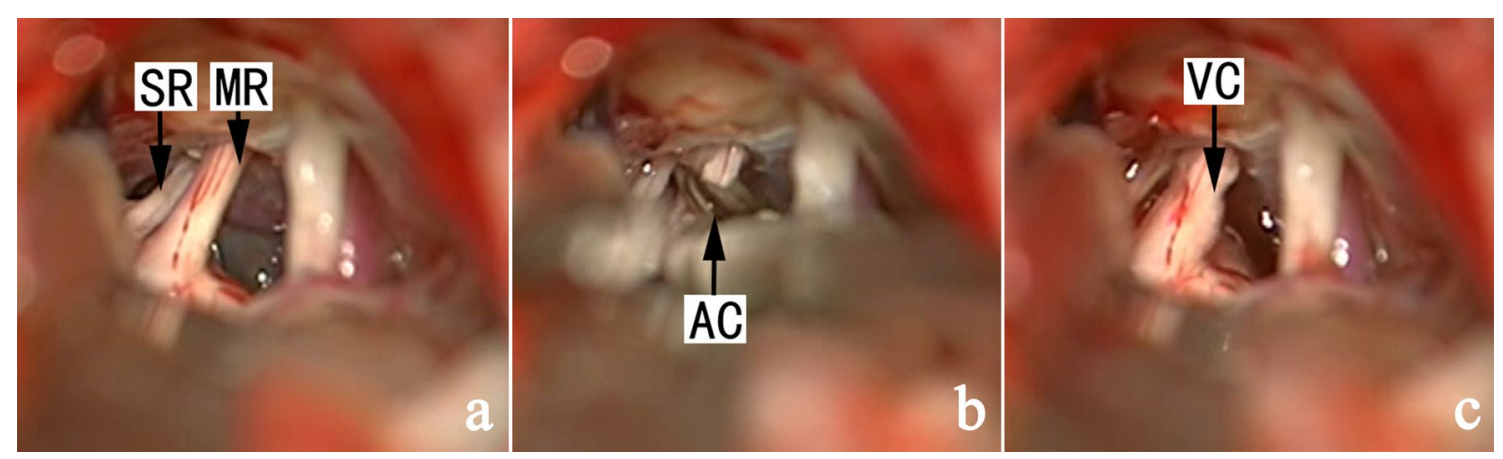

Fig. 1 A representative case with no OVs and separate sensory vs. motor trigeminal nerve root. SR: sensory root of trigeminal nerve, MR: motor root of trigeminal nerve, AC: aneurysm clip, VC: vestige of clamp at the sensory root of trigeminal nerve
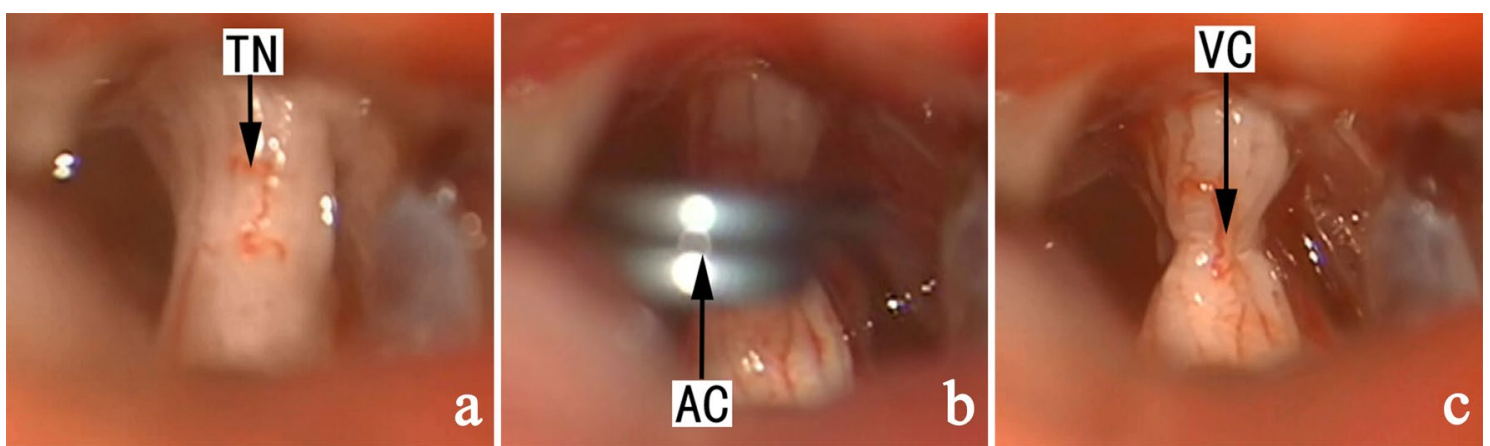

Fig. 2 A representative case with no OVs and united sensory and motor roots. TN: trigeminal nerve, AC: aneurysm clip, VC: vestige of clamp at the whole trigeminal nerve 

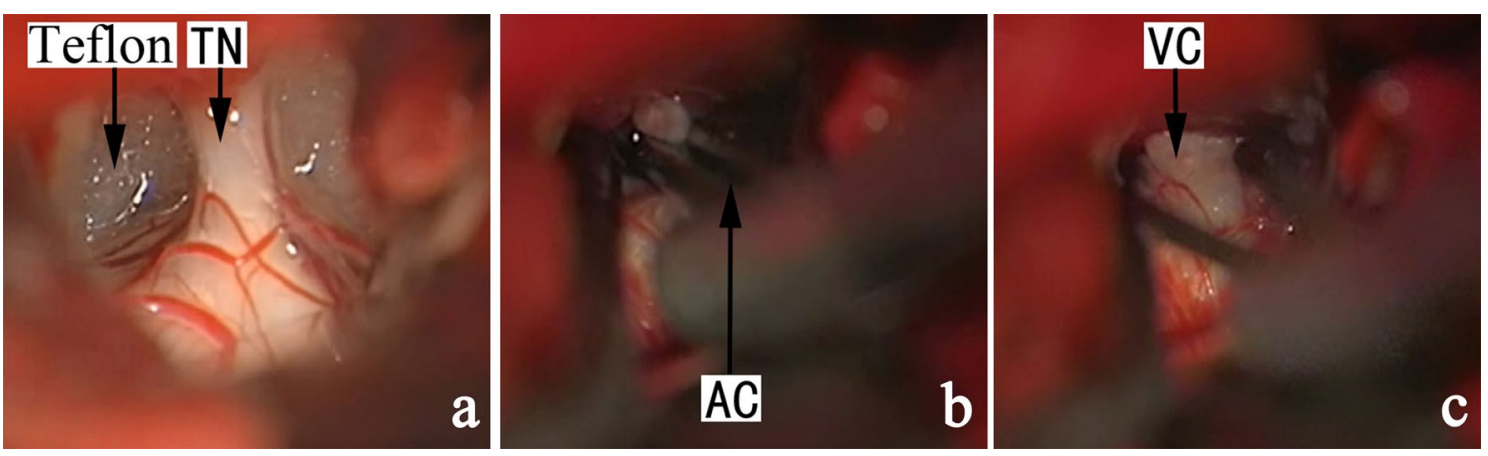

Fig. 3 A representative case of recurrent TGN, with no OVs and united sensory and motor root. TN: trigeminal nerve, AC: aneurysm clip, Teflon: Teflon pad, VC: vestige of clamp at the whole trigeminal nerve

Table 1 Demographic and baseline characteristics of the patients

\begin{tabular}{ll}
\hline & Cohort size $(\mathbf{n}=\mathbf{2 6})$ \\
\hline Female sex, no. (\%) & $15(57.7 \%)$ \\
Age (y), median (range) & $64(33-81)$ \\
Disease duration (y), median (range) & $3.25(0.5-14.0)$ \\
Affected side, no. (\%) & \\
$\quad$ Right only & $14(53.8 \%)$ \\
$\quad$ Left only & $12(46.2 \%)$ \\
Territory involved & \\
V1 & 1 \\
V2 & 3 \\
V3 & 5 \\
V1 + V2 & 2 \\
V2 + V3 & 12 \\
V1 + V2 + V3 & 3 \\
\hline
\end{tabular}

\section{Results}

A total of 26 subjects (median age: 64 years; 15 women) were included in the analysis. Demographic and clinical characteristics are shown in Table 1. Among the 26 patients, 5 received previous MVD for TGN. The median disease duration was 3.25 years (range: $0.5-$ 14.0). The most common territory was $\mathrm{V} 2+\mathrm{V} 3$. The Barrow Neurological Institute (BNI) pain scores were IV in 9 cases, and V in the remaining 17 cases [6].

In 16 out of the 26 cases, neurapraxia was completed in one session. In the remaining 10 cases, the process had to be temporarily suspended and repeated due to bradycardia $(n=7)$ or hypertension $(n=3)$. No severe peri-operative complications (e.g., intracranial hemorrhage, intracranial infection and cerebrospinal fluid leakage) occurred. Post-operative complications included transient hemifacial numbness $(\mathrm{n}=26$; $100 \%)$, herpes labialis $(n=9 ; 34.6 \%)$, masseter weakness
Table 2 Post-operative complications

\begin{tabular}{lc}
\hline Complications & $\begin{array}{c}\text { Cohort size } \\
(\mathbf{n = 2 6 ) ( \% )}\end{array}$ \\
\hline Hemifacial numbness & $26(100)$ \\
Herpes labialis & $9(34.6)$ \\
Masseter weakness & $8(30.7)$ \\
Hemifacial formication & $2(7.7)$ \\
Blunted corneal reflex & $2(7.7)$ \\
\hline
\end{tabular}

$(\mathrm{n}=8 ; 30.7 \%)$, hemifacial formication $(\mathrm{n}=2 ; 7.7 \%)$ and blunted corneal reflex $(n=2 ; 7.7 \%)$ (Table 2). Majority of the complications lasted for 3-6 months and eventually dissipated, but facial formication persisted until the last follow-up in both cases ( 2 and 3 years from the surgery, respectively).

Immediate and complete pain relief (BNI pain score of I without any medication) was achieved in all 26 cases. Within a median follow-up of 3.0 years (range: 1-6 years), recurrence occurred in 3 (11.5\%) patients. The time from surgery to relapse was 1,1 , and 1.5 years, respectively. The BNI pain score was II, II and III with carbamazepine treatment in the 3 patients with relapse. The remaining 23 patients were medication-free.

\section{Discussion}

Vascular compression of the trigeminal nerve root is the leading cause of classic TGN. Other causes include focal arachnoid thickening, adhesion, cerebello-pontine angle tumors, inflammation, multiple sclerosis, brainstem infarction, and arteriovenous malformations [7, 8]. In patients who did not respond to or could not tolerate pharmacological treatments, MVD is the first choice regardless of the presence or absence of NVC as determined by MRI due to the low sensitivity of MRI in detecting NVC [9]. In a small percentage of the patients, NVC 
could not be identified despite of complete dissection of entire length of the trigeminal nerve root. In addition, complete dissection of the trigeminal nerve in recurrent cases after previous MVD is often difficult, if not impossible, due to adhesion $[10,11]$.

Partial sensory rhizotomy (PSR) is one option in patients with no identifiable NVC. In a study of 83 cases, Young et al. reported 15\% rate of severe complications (complete loss of sensory function and corneal ulcer) [12]. A literature review of 10,493 patients undergoing a variety of surgical treatments for trigeminal neuralgia also supported the high rate of severe complication with PSR [13]. As a result, PSR has been practically abandoned in clinical practice. Nerve combing is another option in TGN patients with no identifiable NVC, but is associated with relatively high rate of 5 -year recurrence (approximately 40\%) [14-16].

Percutaneous balloon compression (PBC) at the site of the trigeminal Gasserian ganglion is currently recommended as the first-choice extracranial treatment of TGN [17]. The physiological basis of $\mathrm{PBC}$ is the higher sensitivity of the larger pain fibers in the trigeminal nerve to physical damages compared to smaller fibers that transmit other sensory input and the afferent fibers [18]. Despite of the advantage of PBC, MVD is the treatment of choice in patients with TGN regardless of the presence or absence of NVC as determined by pre-operative MRI. In other words, PBC is typically used in patients who failed MVD treatment, and must be conducted separately $[3,4]$.

Cheng et al. used bipolar electrocoagulation tip to produce neurapraxia in 28 patients without OV. 20 patients (71.4\%) achieved immediate complete pain relief. With a median follow-up of 46 months (range: 8-60 months), the recurrence rate was $38.4 \%$, and only 13 patients (46.4\%) remained pain-free without medication during the follow-up. Four patients (14.3\%) developed permanent facial numbness [19]. Revuelta-Gutierrez et al. used bipolar electrocoagulation tips to produce neurapraxia to the trigeminal nerve root in 21 patients, and achieved immediate complete pain relief in all 21 patients. Recurrence rate was $14.8 \%$ at $12-36$ months and $43.2 \%$ at 48 months. Permanent hypoesthesia was present in 6 patients $(28.6 \%)$, whereas transient loss of corneal reflex was observed in 1 patient (4.8\%). Motor function of the trigeminal nerve was intact in all patients [6].

In the current study, we achieved $100 \%$ immediate complete pain relief with acceptable complications in 26 patients with no identifiable NVC during MVD. Within a median of 3-year follow-up, the recurrence rate was $11.5 \%$. In our opinion, these encouraging results reflect consistent degree of damage to the sensory fibres of the trigeminal nerve due to the use of consistent force $(90 \mathrm{~g})$ and 40-s clipping duration. Whether this method could be developed as a standardizable approach requires further study in different settings.

From a surgical viewpoint, the trigeminal nerve root must be completely exposed to reveal possible OVs. If possible, only the sensory root should be clipped. Also, duration of the clipping is essential. In a pilot series that consisted of 3 cases, we clipped the trigeminal nerve root for $2.5 \mathrm{~min}$, and unfortunately, all 3 patients developed severe hemifacial numbness and masseter weakness. The protocol that we have been using since 2015 is clipping for $40 \mathrm{~s}$ if the procedure could be completely in a single attempt, and for a total of $60 \mathrm{~s}$ if the procedure must be suspended temporarily and repeated due to bradycardia or hypertension.

In addition to the retrospective nature, the current study is limited by the relatively small sample size and the relatively short follow-up (median at 3 years). Also, 3 patients who were lost to follow-up were not included in the analysis. It is likely that these 3 patients experienced relapse or complications but chose not coming back to us. This could produce some bias to our results. The follow-up was conducted via telephone in some patients, and not based on office visit, adding another layer of limitation to the current study. Having said that, we believe that the key results are solid since recurrence and majority of the complications are sensory abnormalities without standard objective examinations.

\section{Conclusions}

Neurapraxia using a Yasargil temporary titanium aneurysm clip is safe and effective in patients with classic TGN but no identifiable NVC during MVD. The advantage of this method include: (1) potential wider use since the damage is standardizable; (2) no need to schedule a second surgery.

\section{Abbreviations}

MVD: Microvascular decompression; TGN: Trigeminal neuralgia; OVs: Offending vessels; CSF: Cerebrospinal fluid; BNI: The Barrow Neurological Institute; PSR: Partial sensory rhizotomy; PBC: Percutaneous balloon compression.

\section{Acknowledgements \\ Not applicable.}

\section{Authors' contributions}

Conception and design: JY; drafting the article: JL and MZ; reviewed the submitted version of manuscript: YW; administrative: SCK. All authors read and approved the final manuscript.

\section{Funding}

This work was supported by the National Natural Science Foundation of China (\#81671201 received by Jia Yin and \#81871598 received by Yuhai Wang).

\section{Availability of data and materials}

The datasets used and/or analysed during the current study are available from the corresponding author on reasonable request. 


\section{Declarations}

\section{Ethics approval and consent to participate}

The current study was approved by the Ethics Committee of Shanghai Tenth People's Hospital (approval \#: CPPRB1). Informed consent was waived due to the nature of the study.

\section{Consent for publication}

Not applicable.

\section{Competing interests}

The authors declare that they have no competing interests.

\section{Author details}

${ }^{1}$ Department of Neurosurgery, Shanghai Tenth People's Hospital, Tongji University, No. 301 Yanchang Road, Shanghai 200072, China. ${ }^{2}$ Department of Neurosurgery, Bengbu First People's Hospital, No. 229 Tushan Road, Bengbu 23000, Anhui, China. ${ }^{3}$ Department of Neurosurgery, 904 Hospital of PLA, No. 101 North Xingyan Road, Wuxi 214044, Jiangsu, China. ${ }^{4}$ Shanghai Key Laboratory of Brain Functional Genomics, Key Laboratory of Brain Functional Genomics Ministry of Education, School of Psychology and Cognitive Science, East China Normal University, No. 3663 North Zhongshan Road, Shanghai 200062, China. ${ }^{5}$ Shanghai Key Laboratory of Magnetic Resonance, East China Normal University, No. 3663 North Zhongshan Road, Shanghai 200062, China. ${ }^{6}$ NYU-ECNU Institute of Brain and Cognitive Science at NYU Shanghai, No. 3663 North Zhongshan Road, Shanghai 200062, China.

Received: 7 May 2021 Accepted: 4 January 2022

Published online: 11 January 2022

\section{References}

1. Debta P, Sarode G, Sarode S, Gadbail A, Debta FM, Swain SK, et al. Natural history of trigeminal neuralgia-A hospital-based retrospective study. Oral Dis. 2020;26:647-55.

2. Jannetta PJ. Arterial compression of the trigeminal nerve at the pons in patients with trigeminal neuralgia. J Neurosurg. 1967;26(Suppl):159-62.

3. Cruccu G, Gronseth G, Alksne J, Argoff C, Brainin M, Burchiel K, et al. AAN-EFNS guidelines on trigeminal neuralgia management. Eur J Neurol. 2008;15:1013-28.

4. Bendtsen L, Zakrzewska JM, Abbott J, Braschinsky M, Di Stefano G, Donnet $A$, et al. European Academy of Neurology guideline on trigeminal neuralgia. Eur J Neurol. 2019;26:831-49.

5. Revuelta-Gutiérrez R, López-González MA, Soto-Hernández JL. Surgical treatment of trigeminal neuralgia without vascular compression: 20 years of experience. Surg Neurol. 2006;66:32-6.

6. Rogers CL, Shetter AG, Fiedler JA, Smith KA, Han PP, Speiser BL. Gamma knife radiosurgery for trigeminal neuralgia: the initial experience of The Barrow Neurological Institute. Int J Radiat Oncol Biol Phys. 2000;47:1013-9.

7. Thomas KL, Vilensky JA. The anatomy of vascular compression in trigeminal neuralgia. Clin Anat. 2014;27:89-93.

8. Sindou M, Howeidy T, Acevedo G. Anatomical observations during microvascular decompression for idiopathic trigeminal neuralgia (with correlations between topography of pain and site of the neurovascular conflict). Prospective study in a series of 579 patients. Acta Neurochir (Wien). 2002;144:1-12.

9. Antonini G, Di Pasquale A, Cruccu G, Truini A, Morino S, Saltelli G, et al. Magnetic resonance imaging contribution for diagnosing symptomatic neurovascular contact in classical trigeminal neuralgia: a blinded casecontrol study and meta-analysis. Pain. 2014;155:1464-71.

10. Lee A, McCartney S, Burbidge C, Raslan AM, Burchiel KJ. Trigeminal neuralgia occurs and recurs in the absence of neurovascular compression. J Neurosurg. 2014;120:1048-54. d

11. Ishikawa M, Nishi S, Aoki T, Takase T, Wada E, Ohwaki H, et al. Operative findings in cases of trigeminal neuralgia without vascular compression: proposal of a different mechanism. J Clin Neurosci. 2002;9:200-4.

12. Young JN, Wilkins RH. Partial sensory trigeminal rhizotomy at the pons for trigeminal neuralgia. J Neurosurg. 1993;79:680-7.
13. Tatli M, Satici $O$, Kanpolat $Y$, Sindou M. Various surgical modalities for trigeminal neuralgia: literature study of respective long-term outcomes. Acta Neurochir (Wien). 2008;150:243-55.

14. Jie H, Xuanchen Z, Deheng L, Kun G, Fengyang X, Xiang C, et al. The longterm outcome of nerve combing for trigeminal neuralgia. Acta Neurochir (Wien). 2013;155:1703-8.

15. Ko AL, Ozpinar A, Lee A, Raslan AM, McCartney S, Burchiel KJ. Long-term efficacy and safety of internal neurolysis for trigeminal neuralgia without neurovascular compression. J Neurosurg. 2015;122:1048-57.

16. Urculo E, Elua A, Arrazola M, Torres P, Torres S, Undabeitia J. Trigeminal root massage in microsurgical treatment of trigeminal neuralgia patients without arterial compression: when, how and why. Neurocirugia (Astur). 2020;31:53-63.

17. Mullan S, Duda EE, Patronas NJ. Some examples of balloon technology in neurosurgery. J Neurosurg. 1980;52:321-9.

18. Peris-Celda M, Graziano F, Russo V, Mericle RA, Ulm AJ. Foramen ovale puncture, lesioning accuracy, and avoiding complications: microsurgical anatomy study with clinical implications. J Neurosurg. 2013;119:1176-93.

19. Cheng J, Lei D, Zhang H, Mao K. Trigeminal root compression for trigeminal neuralgia in patients with no vascular compression. Acta Neurochir (Wien). 2015;157:323-7.

\section{Publisher's Note}

Springer Nature remains neutral with regard to jurisdictional claims in published maps and institutional affiliations.
Ready to submit your research? Choose BMC and benefit from:

- fast, convenient online submission

- thorough peer review by experienced researchers in your field

- rapid publication on acceptance

- support for research data, including large and complex data types

- gold Open Access which fosters wider collaboration and increased citations

- maximum visibility for your research: over 100M website views per year

At BMC, research is always in progress.

Learn more biomedcentral.com/submissions 